\title{
CARACTERÍSTICAS SÓCIO-DEMOGRÁFICAS E REPRODUTIVAS DE ADOLESCENTES ATENDIDAS NO PÓS-PARTO NO INSTITUTO DA CRIANÇA DA UNIVERSIDADE DE SÃO PAULO ${ }^{+}$
}

\author{
SOCIO-DEMOGRAPHIC AND REPRODUCTIVE CHARACTERISTICS OF \\ ADOLESCENT MOTHERS WHO ATTENDED AN INPATIENT CLINIC LINKED \\ TO THE ADOLESCENT UNIT OF A PUBLIC HOSPITAL
}

Nicole O Machado*

Maria I. Saito*

Sophia C Szarfarc ${ }^{* *}$

\begin{abstract}
Machado NO, Saito MI, Szarfarc SC. Características sócio-demográficas e reprodutivas de adolescentes atendidas no pós-parto no Instituto da Criança da Universidade de São Paulo. Rev Bras Crescimento Desenvol Hum. 2007; 17(3):01-07.
\end{abstract}

\begin{abstract}
Resumo: Tendo em vista os problemas inerentes à gravidez na adolescência e a importância de direcionar ações de saúde para essa população a Unidade de Adolescentes do Instituto da Criança do Hospital das Clínicas da USP estendeu o atendimento, oferecendo atenção integral para mães adolescentes e seus filhos que freqüentaram o pré-natal na Disciplina de Obstetrícia da FMUSP. Este estudo visou identificar algumas características do modo de vida das mães adolescentes que freqüentam esse serviço. A população amostral foi constituída pelas 81 jovens que freqüentavam o ambulatório no período de estudo. Verificou-se que 76,5\% delas não planejaram a gestação. Mesmo assim, com o apoio familiar e da instituição, planejavam mais filhos em data futura. A maioria delas convivia com o companheiro que, algumas vezes, assumiu também a chefia da família da adolescente. Grande parte delas, 54,3\% abandonou os estudos e, das 59 mães que trabalhavam, 38 deixaram de fazê-lo. As atividades de lazer foram modificadas e cerca de metade das jovens deixou de participar de qualquer atividade de lazer. O estudo permite ressaltar as repercussões da maternidade na vida das jovens e evidencia a importância do pré-natal e de serviços que proporcionem atendimento pós-parto, enfocando a saúde de uma forma global, beneficiando as adolescentes e seus filhos.
\end{abstract}

Palavras-chave: Mães adolescentes. Estilo de vida. Programas de atendimento pós-natal.

\section{INTRODUÇAO}

A adolescência caracteriza-se por mudanças dinâmicas em todas as esferas do desenvol- vimento humano. Nessa fase da vida ocorrem crescimento e desenvolvimento intensos que são fortemente influenciados por fatores genéticos e ambientais como os de nível sócio-econômico.

* Instituto da Criança do Hospital das Clínicas da Faculdade de Medicina da Universidade de São Paulo

** Departamento de Nutrição - Faculdade de Saúde Pública / Universidade de São Paulo.

Endereço: Av Dr. Arnaldo, 715 - Cerqueira César - São Paulo-SP.CEP: 01246-904

Instituição onde o trabalho foi realizado: Faculdade de Saúde Pública / Departamento de Nutrição

Autor responsável para envio de correspondência: Sophia Cornbluth Szarfarc

Endereço: Av Dr. Arnaldo, 715 CEP: 01246-904 Cerqueira Cesar São Paulo - SP.

+ Artigo baseado na dissertação de mestrado de Nicole O Machado, "Caracterização nutricional de uma população de mães adolescentes”. Curso de Pós-Graduação em Saúde Pública, área Nutrição da Faculdade de Saúde Pública da Universidade de São Paulo. 
A adolescência, que se estende dos 10 aos 19 anos $^{1}$, é o período da vida em que se estabelecem novas relações do indivíduo com o meio social, com outros adolescentes, mudanças nas relações com a família, sendo relevante nessa época a preocupação com a imagem corporal ${ }^{2}$.

Do ponto de vista psicológico e social ocorrem mudanças importantes que marcam a passagem para o mundo adulto. Questões relacionadas à busca de si mesmo e da identidade, separação progressiva dos pais, vinculação a um grupo, atitudes sociais reivindicatórias, desenvolvimento do pensamento abstrato e evolução da sexualidade, dentre os que compõem a Síndrome da Adolescência Normal ${ }^{3}$.

Escolhas educacionais e profissionais, relações interpessoais e de cidadania são ao mesmo tempo preocupação e responsabilidade do adolescente que se intensificam quando ocorre uma gravidez sempre precoce e, na maior parte das vezes, não planejada.

É indiscutível que uma das mudanças mais importantes que ocorrem nessa etapa da vida está relacionada ao início da atividade sexual e, conseqüentemente, risco de uma gravidez. É cada vez mais precoce e frequente a atividade sexual entre jovens e a gravidez, nessa fase da vida, é sempre impactante pois, sem ter alcançado maturidade emocional e independência financeira, a mulher terá a sobrecarga de criar um filho.

Especialmente quando ocorre entre a população de baixo nível sócio-econômico, há evidências de que além dos problemas biológicos e psicossociais para o binômio mãe/filho ${ }^{4,5}$, que são mais importantes quanto mais jovem for a mulher, a adolescente terá menos oportunidade de estudar e conquistar um espaço profissional do que as mulheres que adiam a maternidade para a idade adulta ${ }^{6,7,8}$.

Reconhecendo os problemas inerentes à gestação na adolescência e a carência de serviços específicos para esse grupo populacional, a Unidade de Adolescentes do Instituto da Criança (UA - ICr) do Hospital das Clínicas da Faculdade de Medicina da Universidade de São Paulo, iniciou, em 1996, atendimento às mães adolescentes que freqüentaram o pré-natal da Instituição, paralelamente à atenção aos seus filhos visando o resgate do seu projeto de vida, incentivo a maternagem e a prevenção de nova gestação.

Este estudo visou identificar algumas características do modo de vida das jovens atendidas no pós natal da UA - ICR.

\section{METODOLOGIA}

Este estudo foi aprovado pelo Comitê de Ética do Hospital das Clínicas e teve o consentimento livre e esclarecido da população de estudo.

Participaram do estudo as 81 jovens que constituíam o universo de mães em atendimento. Da entrevista utilizada para coleta de dados constavam questões relativas à idade por ocasião do parto, escolaridade, trabalho, lazer, amamentação, peso e estatura da criança ao nascer; relativas à família: situação conjugal e tipo de união; identificação e escolaridade do chefe da família.

\section{RESULTADOS}

Foram entrevistadas 81 adolescentes sendo que 68 delas eram primigestas. Merece destaque o fato de que 8 delas referiram gestações anteriores àquela que motivou sua admissão no programa especial da UA - ICr, e 5 estarem grávidas novamente por ocasião da pesquisa.

A Tabela 1 apresenta a inserção das jovens na família.

Tabela 1 Distribuição da população de mães segundo núcleo familiar com quem mora. UA - ICr, 2001.

\begin{tabular}{l|ll}
\hline Com quem mora & $\mathrm{N}^{\mathrm{o}}$ & $\%$ \\
\hline Companheiro & 36 & 44,4 \\
Família de origem e comp anheiro & 11 & 13,6 \\
Família do companheiro & 2 & 2,5 \\
Família de origem & 30 & 37 \\
Sozinha com o filho & 2 & 2,5 \\
\hline
\end{tabular}

Pode-se observar a presença do companheiro junto à adolescente na maior parte dos casos, independentemente do tipo e tempo de união e, mesmo, do planejamento da gravidez, uma vez que $76.5 \%$ (62) das jovens não haviam planejado a gravidez.

A chefia da família acompanha, em parte 
a situação do núcleo familiar (Tabela 2)

Tabela 2 - Distribuição da população de mães segundo relação com o chefe da família, UA - ICr, 2001

\begin{tabular}{l|ll}
\hline Chefe da família & $\mathrm{N}^{\mathrm{o}}$ & $\%$ \\
\hline Companheiro & 41 & 50.6 \\
Pai/Mãe/Avô & 36 & 44.5 \\
Sogro & 3 & 3.7 \\
A própria adolescente & 1 & 1.2 \\
\hline
\end{tabular}

Das 51 jovens que referiram união consensual ou legal, 28 haviam consolidado a união há menos de dois anos, o que sugere que a união tenha ocorrido, possivelmente, em função da gestação ou do nascimento do filho.

A Tabela 3 apresenta a situação de escolaridade da jovem e do chefe da família.

Tabela 3 - Distribuição da população da mãe e do chefe da família segundo escolaridade. UA-ICr. 2001.

\begin{tabular}{l|ll}
\hline Nível escolar & $\begin{array}{l}\mathrm{Mãe} \\
\mathrm{N}^{\mathrm{o}}(\%)\end{array}$ & $\begin{array}{l}\text { Chefe } \\
\mathrm{N}^{\mathrm{o}} *(\%)\end{array}$ \\
\hline Analfabeto & 0 & $2(2.6)$ \\
$1^{\circ}$ grau incompleto & $32(39.5)$ & $41(53.9)$ \\
$1^{\circ}$ grau completo & $11(13.6)$ & $5(6.6)$ \\
$2^{\circ}$ grau incompleto & $17(21.0)$ & $11(14.5)$ \\
$2^{\circ}$ grau completo ou mais & $21(25.9)$ & $17(22.4)$ \\
\hline * - 5 sem informação
\end{tabular}

Embora o conhecimento da renda familiar seja um importante indicador da possibilidade de aquisição e utilização de serviços essenciais à saúde, praticamente 50\% (39) das jovens não souberam referir o dado. Dentre as que informaram a renda familiar, $55 \%$ recebia menos do que 5 salários mínimos ao mês. Esse rendimento é compatível com a baixa escolaridade tanto do chefe da família como da própria adolescente e também concordante com o tipo de trabalho desenvolvido por aquelas poucas jovens que exerciam alguma atividade laborial. As ocupações exercidas não exigiam especialização e, na maioria das vezes, estavam enquadradas em atividades de trabalho informal: limpeza, auxiliar de costura, ajudante de recepção, vendedora etc. No momento da entrevista, das 81 jovens, $35,8 \%$ nunca trabalhou, outras 38 já haviam trabalhado mas apenas 14 estavam trabalhando.
Das que haviam trabalhado, 11 foram despedidas por não corresponderem às funções. $\mathrm{A}$ maior parte das outras parou de trabalhar em função do bebê (gravidez, casamento, cuidados com a criança). Diferente do que ocorre com mulheres em trabalhos informais, não houve nenhuma referência à perda de emprego por conta da gravidez.

A Tabela 4 apresenta a idade das adolescentes por ocasião do parto e sua associação com o peso ao nascer.

Tabela 4 - Associação da idade cronológica no parto e peso do filho (PN) ao nascimento, UA - ICR, 2001.

\begin{tabular}{l|ccc}
\hline Idade (anos) & PN $<2500 \mathrm{~g}$ & $\mathrm{PN} \geq 2500 \mathrm{~g}$ & Total \\
\hline$<17$ & 7 & 16 & 23 \\
$17-19$ & 35 & 34 & 69 \\
Total & 42 & 39 & 81 \\
\hline \multicolumn{4}{l}{$\mathrm{X}^{2}=5,8 \mathrm{p}<0.05$}
\end{tabular}

Com relação aos cuidados com o bebê, chama a atenção que $70 \%$ das adolescentes amamentaram seus filhos por 4 meses ou mais o que reflete a importância da atenção recebida no pré-natal e na UA - ICr. No entanto, com relação à nova gestação, a atenção dada às jovens foi incapaz de cumprir seu objetivo de prevenir nova gestação e 5 das adolescentes estavam grávidas novamente.

A Tabela 5 apresenta as atividades de lazer desenvolvidas pelas mães, antes, durante e após o parto.

Tabela 5 - Distribuição porcentual da população de jovens segundo tipo de atividade de lazer. UA-ICr, 2001

\begin{tabular}{l|ccc}
\hline ATIVIDADE (N=81) & \multicolumn{3}{|c}{ ANTES DURANTE DEPOIS } \\
\hline Festas, bailes,danceterias & 46,9 & 13,6 & 11,1 \\
Parques, praças & 32,1 & 19,8 & 24,7 \\
Shopping & 30,9 & 19,8 & 22,2 \\
Barzinhos, restaurantes & 22,2 & 8,6 & 13,6 \\
Visitas a parentes, amigos & 13,6 & 20,1 & 27,2 \\
Outros & 27,2 & 13,6 & 8,6 \\
Nenhuma & 25,9 & 45,7 & 45,7 \\
\hline
\end{tabular}

\section{DISCUSSÃO E COMENTÁRIOS}

Mães adolescentes e seus filhos represen- 
tam uma população de maior risco ${ }^{9,10,11 .}$ Embora alguns autores ${ }^{12}$ sugiram que o risco biológico decorrente da gravidez na adolescência seja controvertido, podendo ser minorado por uma atenção pré-natal específica, relatam também que o risco causado pela situação sócio-econômica e idade materna no desenvolvimento do bebê, é consistente.

Também o Committee on Adolescence ${ }^{13}$ refere que mães adolescentes tem menos chances de estudar e de conseguir boas oportunidades de trabalho que seu pares que adiam a maternidade para a idade adulta. Destaca ele que o nível educacional tem mais impacto que a idade biológica no desenvolvimento cognitivo e de saúde de seus filhos.

Conhecendo os problemas inerentes à gravidez na adolescência, o aumento na demanda de atendimento específico para essa população e a ausência de serviços públicos de saúde destinados a mães adolescentes, a Unidade de Adolescentes do Instituto da Criança do Hospital das Clinicas da Faculdade de Medicina da Universidade de São Paulo iniciou, em 1996, atendimento ambulatorial para filhos de mães adolescentes que freqüentaram o pré-natal específico para adolescentes da Disciplina de Obstetrícia, estendendo a elas a atenção de acompanhamento e orientação. Por ocasião da consulta de puericultura, as jovens recebiam orientações destinadas tanto a fortalecer o vínculo mãe/filho, como de incentivo ao planejamento familiar e à prevenção de nova gestação e, principalmente, visando a estimular a retomada do projeto de vida materno. Com relação ao último parto, motivo pelo qual freqüentavam o serviço e foram incluídas nesta pesquisa, observou-se que $76,5 \%$ jovens não haviam planejado a gravidez. Dados semelhantes foram encontrados por outros autores ${ }^{14,15}$. Embora não tendo planejado a gravidez, 45,7\% pretendia ter mais filhos, preferindo porém adiar o evento por alguns anos. Destacamos que, no entanto, 32\% delas não utilizava nenhum método para evitá-la. Os métodos preventivos mais utilizados foram os anticoncepcionais orais para $51 \%$ e, a camisinha, para $27 \%$ das jovens. $\mathrm{O}$ estudo chamou a atenção para o fato de que, embora esta população tenha vivenciado a ex- periência de uma gestação durante a adolescência, boa parte dela manifestou desejo de nova gravidez, num futuro distante, o que sugere uma satisfação com o evento e estabilidade emocional decorrentes, provavelmente, do apoio recebido da família e do suporte da Instituição que freqüentaram e da que continuam freqüentando com seus filhos. O atendimento da população em um serviço de pré-natal especializado pode ter se refletido na menor proporção de récem-nascidos de baixo peso (15\%), e no maior período de aleitamento, quando comparado a outros estudos com adolescentes ${ }^{10,16,17}$. Das adolescentes do estudo 28 estavam amamentando e 8 nunca amamentaram. Dentre as que amamentaram 21 $(46,7 \%)$ mantiveram o aleitamento por um período igual ou maior a 4 meses sendo que 14 delas mantiveram a amamentação por 6 meses ou mais. Considerando aquelas que já haviam interrompido o aleitamento, menos da metade proporcionou o período mínimo recomendado pela $\mathrm{WHO}^{18}$. Apesar disto, destacamos os achados pois estes diferem dos de outros estudos ${ }^{19,20}$ que mostram diferenças significantes na prática do aleitamento entre mães adolescentes onde apenas $21 \%$ delas amamentaram até o $4^{\circ} \mathrm{mês}^{21}$, quando comparadas a mães mais velhas. Radius e Joffe ${ }^{19}$ sugerem em seu estudo que as adolescentes que optaram pelo aleitamento são aquelas que distinguiram os benefícios associados a essa prática. Estes achados sugerem que programas educativos são benéficos, enfocando, enumerando e trabalhando os benefícios potenciais do aleitamento.

O fato das jovens deste estudo terem frequentado um serviço de pré-natal especializado onde receberam orientações sobre aleitamento materno e seus benefícios, alimentação da nutriz e outros cuidados relacionados à gestante e seus filhos, pode ter influenciado estes resultados. Dentre elas, $87,7 \%$ referiram haver recebido orientação sobre aleitamento materno. Das que receberam orientações, $71,6 \%$ citaram a equipe do pré-natal como responsável, ou seja, estas jovens também beneficiaram seus conceptos, neste aspecto, com o programa deste serviço, como em outros estudos ${ }^{22}$ Entretanto, aparentemente, os benefícios não se estenderam à pre- 
venção de novas gestações, uma vez que 5 delas estavam grávidas novamente. Resultados semelhantes foram encontrados por Aquino et $\mathrm{al}^{8}$, que referem que apesar de mais de $52 \%$ das jovens manifestarem desejo de não engravidar ou de adiar a maternidade, $68,6 \%$ não faziam uso de contraceptivos.

Diferente do que é descrito para adolescentes grávidas, com uma única exceção, a população de estudo recebeu o apoio do companheiro e/ou da família de origem diante da gravidez. Metade delas referiu o companheiro como sendo o chefe da família sendo que em 5 casos este assumiu, também, a responsabilidade pelo sustento da família da mulher (Tabelas 1 e 2).

A baixa escolaridade encontrada entre as adolescentes atendidas no UA - ICr, é característica da população que frequienta serviços públicos de saúde. Das 81 jovens entrevistadas, 44 já haviam interrompido os estudos (Tabela 3). A situação conjugal (companhei ro, gestação, bebê) foi a razão mais citada para o abandono da escola, porém, parte delas (25\%) declarou "não estar afim" de freqüentar uma escola.

Como mostra a Tabela 3, mais de metade da população de adolescentes não completaram o primeiro grau escolar o que, por sua vez, sugere que o abandono escolar foi anterior à gestação, uma vez que a idade média das mães, no momento da entrevista era de 17 anos e 9 meses, cabendo lembrar que a baixa escolaridade é considerada com fator de risco para gestação, nesta faixa etária. Mesmo com essa baixa escolaridade, a população atendida no UA - ICr encontra-se em situação privilegiada em relação ao observado entre mulheres adolescentes ou não, que freqüentam serviços públicos de saúde brasileiros. Spinelli et $\mathrm{al}^{23}$ verificaram que apenas 5\% das mães, de uma amostra de 2600 mulheres, que freqüentavam serviços públicos de saúde de 12 capitais brasileiras, haviam completado o $1^{\circ}$ grau escolar e ultrapassado esse nível. Dentre essas mulheres apenas $34 \%$ havia completado o primeiro ciclo do $1^{\circ}$ grau, antigo primário. Também Horta et $\mathrm{al}^{21}$ relatam a baixa escolaridade materna, entre mães adolescentes, onde 19,4\% tinham menos de 5 anos de escolaridade. Alguns estudos sugerem, como descrito para esta população, que ter filhos na adolescência aumenta a chance de afastamento da escola e do trabalho. Alguns autores ${ }^{8,23}$, concordam com essa afirmação e acrescentam que a baixa escolaridade diminui a oportunidade de uma profissionalização e conseqüente obtenção de um trabalho seguro e bem remunerado. Souza ${ }^{22}$ faz uma afirmação ainda mais contundente quando refere que a maternidade na adolescência é função da pobreza e guarda sinergismo com ela, perpetuando e/ou agravando a situação sócioeconômica.

O tipo de ocupação referido pelas 52 adolescentes que trabalhavam e/ou trabalharam, sempre muito modesto, mostra nitidamente a influência maléfica decorrente da baixa escolaridade. Se a capacitação deficiente para exercer atividades de trabalho ocorre com a população em geral independente de gênero e idade, ela é muito mais notada entre mães e mães adolescentes. Essa situação vem sendo descrita em várias pesquisas realizadas no Brasil. Marques e Ebrahim ${ }^{24}$, entre outros ${ }^{25}$, destacam o papel da maternidade precoce na interrupção dos estudos e sua conseqüência no desenvolvimento pessoal das jovens.

Os riscos biológicos entre os quais a maior incidência de baixo peso ao nascer na adolescência, especialmente entre as mais jovens, são amplamente discutidos na literatura ${ }^{26,27}$. A Tabela 4 mostra a associação estatisticamente significante entre incidência de baixo peso ao nascer e idade. Diferente, porém, do que seria esperado, as jovens com 17 e mais anos apresentaram proporção maior de nascidos de baixo peso do que aquelas com 16 anos e menos. Esse achado inesperado difere do observado no Brasil ${ }^{27}$, entre mães adolescentes. Mariotoni e Barros ${ }^{26}$, discutindo a gravidez na adolescência como fator de risco para o baixo peso ao nascer, na população brasileira, encontraram o que vem sendo relatado na literatura em geral: incidência maior de baixo peso ao nascer entre as jovens com menos de 17 anos (16,3\%) e menor entre aquelas com 17 anos e mais (12,3\%), diferença essa estatisticamente significante $(a ́<0,05)$.

Por sua vez, na amostra estudada observou-se que a estatura dos filhos das adolescen- 
tes, ao nascimento, foi menor que a esperada, o que pode ser justificado pelo estado nutricional prévio da jovem e ao início tardio ao pré-natal uma vez que a estatura depende da situação nutricional prévia da gestante e, se define no primeiro trimestre de gestação.

É interessante observar a relação das jovens com as atividades de lazer (Tabela 5). A mudança de vida resultou para a maior parte das mulheres em uma não adaptação à nova situação. Boa parte delas não consegue encontrar na nova rotina implantada, quer pela gestação, quer pela presença de um bebê no lar, atividades que sejam desenvolvidas prazeirosamente e, assim, se tornem atividades de lazer. Como mostra a Tabela 5, praticamente a metade delas relatou não ter qualquer atividade de lazer. Passeios em parques e praças, muito citados como atividades de lazer antes e durante a gravidez, passaram a não ser mais vistos como tal após o nascimento do filho, embora devam ter se tornado mais freqüentes. $\mathrm{O}$ fato de haver uma orientação do serviço de saúde para levar o bebê a esses locais, tirou para a jovem, a conotação até então reconhecida como passeio e, portanto, lazer. Raramente sair com o bebê, para realizar atividades domésticas como compras, pagamentos etc era interpretado como lazer. Se por um lado, visitas a parentes e amigos se tornaram muito mais freqüentes, por outro lado, atividades que não permitem a participação de crianças como "baladas", festas, bailes, danceterias e outros se tornaram menos freqüentes, o que foi justificado por motivos diversos: amamentação, tempo, dinheiro e não ter com quem deixar o filho.

Este estudo chama a atenção para o fato de que embora a população estudada tenha vivenciado a experiência de uma gestação na adolescência ela é diferenciada em relação ao suporte familiar e institucional recebido, tendo procurado, freqüientado e se mantido em um atendimento de pré e pós natal vinculados à USP, o que garante a sua qualidade.

Os resultados com relação à prevalência de baixo peso ao nascer e maior tempo de aleitamento também evidenciam a importância de serviços destinados a gestantes que dêem continuidade ao atendimento ao binômio mãe-filho após o parto.

Se, por um lado, a implementação de serviços de saúde semelhantes na rede pública de saúde é desejável, por outro lado, a orientação precoce dos jovens sobre sexualidade e prevenção de gestação é fundamental para minimizar a ocorrência precoce e indesejável de gestações. Cabe lembrar que somente a educação sexual não conduzirá às mudanças desejadas posto que se faz relevante a oportunidade de realização de outro projeto de vida, que não envolva a maternidade precoce.

\begin{abstract}
Taking into account the inherent problems concerning adolescent pregnancy and the importance of implementing health actions targeted at that population, this paper aims to identify social characteristics of adolescent mothers who were followed up, during the prenatal period, by the Discipline of Obstetrics of the School of Medicine of the University of São Paulo, linked to the Adolescent Unit of Hospital das Clínicas. The sample population was composed of the 81 mothers who attended that Unit during the study period. Information concerning personal characteristics was collected by a survey. It was verified that $76.5 \%$ of the young mothers had not intended to become pregnant, but with the support provided by the family and the institution, they were planning to have more babies in the future. The majority of them lived with their partner, who, in some cases, became the head of the adolescent's family. Of the 81 mothers, $39.5 \%$ did not complete elementary school; $54.3 \%$ quitted studying and $49 \%$ did not work. Their leisure activities changed and approximately half of the adolescents stopped participating in any leisure activity, during and after pregnancy. The study emphasizes the importance of prenatal and postnatal care in order to benefit those young women and their children.
\end{abstract}

Keywords: adolescent mothers, lifestyle, postnatal care. 


\section{REFERENNCIAS}

1 Organization Panamericana de la Salud. Fecundidad en la adolescencia: causas, riesgos y opciones. Washington (DC); 1988. (Cuaderno Técnico, 12).

2 Saito, MI. Nutrição In: Secretaria de Estado da Saúde de São Paulo. Adolescência e saúde. $2^{\text {a }}$ Ed.; 1994. p.87-95.

3 Aberastury A, Knobell M La adolescencia normal. Buenos Ayres. Pydós; 1970

4 Correa MGBRM, Coates V. Gravidez. In: Coates V, Françoso LA, Beznos GW. Medicina do Adolescente. São Paulo: Sarvier; 1993.p.259-62.

5 Oliveira MAC, Egry EV. A adolescência enquanto fenômeno social: possibilidades e necessidades de investigação científica em enfermagem. Rev Bras Enferm 1993;46:63-7.

6 Cesar CC, Ribeiro PM, Abreu DMX. Efeito idade ou efeito pobreza?. Mães adolescentes e mortalidade neonatal em Belo Horizonte. Revista Brasileira de Estudos de População vol.17 n.1/2, jan/dez 2000.

7 [BEMFAM] Sociedade Civil Bem- Estar Familiar no Brasil. Brasil: Pesquisa Nacional sobre Demografía e Saude, 1996. Rio de Janeiro;1997.

8 Aquino EML, Knauth D, Bozon M, Almeida MC, Araujo J, Menezes G. Adolescência e reprodução no Brasil: a heterogeneidade dos perfis sociais. Cad. Saúde Pública, Rio de Janeiro, 19(Sup. 2):S377S388, 2003.

9 Worthington RB, Endres J. Nutrition management of adolescent pregnancy: thecnical support paper. J Am Diet Assoc 1989; 89: 105-9.

10 Gama SGN, Szwarwald CL, Leal MC, Filha MM. Gravidez na adolescência como fator de risco para baixo peso ao nascer no Município do Rio de Janeiro, 1996 a 1998. Ver. Saúde Publica 2001;35(1):74-80.

11 Orvos H, Nyirati I, Hadju J, Pal A, Kovacs L. Is adolescent pregnancy associated with adverse perinatal outcome?. J Perinatal Med 1999; 27:199203.

12 Souza RKT, Gotlieb SLD. Probabilidade de morrer no primeiro ano de vida em área urbana da região sul. Revista de Saúde Pública 1993; 76: 445-54.

13 Committee on Adolescence: care of adolescent parents and their children. Pediatrics 1989; 83:13840.

14 Ferraz EA, Ferreira IQ, Soares MP, Morris L. Pesquisa sobre saúde reprodutiva e sexualidade do jovem. Rio de Janeiro, Sociedade Civil Bem-Estar BEMFAM/U.S Department of Health and Human
Services/CDC; 1992. p.75-104: saúde reprodutiva dos jovens.

15 Menezes IHCF. Gestante adolescente: percepção das transformações do corpo. Goiânia; 1998. [Dissertação de Mestrado - Faculdade de Educação da Universidade Federal de Goiás].

16 Mc Anarney ER, Lawrence RA, Aten MJ, Iker HP. Adolescent mothers and their Infants. Pediatrics 1984; 73:358-62.

17 Mariotoni GGB, Barrros FFA. Gravidez na adolescência é fator de risco para baixo peso ao nascer? J Pediatr, Rio 1998; 74:107-13.

18 [WHO] World Health Organization. Innocenti Declaration on the protection, promotion and support of breast feeding. Ecol Food Nutr 1991; 26:271-3.

19 Radius SM, Joffe A. Understanding adolescent mother's feelings about breast -feeding. J Adolesc Health Care 1988; 9: 150-60.

20 Vieira LF, Pinto e Siva JLC, Filho AAB. Amamentação e a alimentação complementar de filhos de mães adolescentes são diferentes das de filhos de mãe adultas? J Pediatr (Rio J) 2003; 79(4):317-24.

21 Horta BL, Victora CG, Gigante DP, santos J, Barros FC. Duração da amamentação em duas gerações. Rev Saúde Pública 2007; 41(1): 13-18.

22 Souza MMC A. maternidade nas mulheres de 15 a 19 anos como desvantagem social. In: Vieira EM, Fernandes MEL, Bailey P, Mckay A, organizadores. Seminário Gravidez na Adolescência, 1998, Rio de Janeiro; Brasil. p.74-91.

23 Spinelli MGN, Marchioni DML, Souza JMP, Souza SB, Szarfarc SC. Fatores de risco para anemia em crianças de 6 a 12 meses no Brasil. Rev Panam Salud Publica 2005;17(2):84-91.

24 Marques NM, Ebrahim MGJ. The antecedents of adolescent pregnancy in a Brazilian squatter community. J Trop Pediatr 1991;37:194-8.

25 Machado NO. Caracterização nutricional de uma população de mães adolescentes. [dissertação]. São Paulo (SP): Faculdade de saúde Pública da Universidade de São Paulo, 2001.

26 Mariotoni GGB, Barros FAAA. Gravidez na adolescência é fator de risco para baixo peso ao nascer? J Pediatr (RJ) 1998;74:107-13.

27 Szarfarc SC, Souza SB, Furumoto RAV, Brunken GS, Assis AMO, Gaudenzi EM et al. A concentração de hemoglobina no primeiro ano de vida da clientela de serviços públicos de saúde do Brasil. Cad Saúde Publica 2004; 20(1):266-74. 